\title{
Interface geometries in 3D multi-material prints by fused filament fabrication
}

\author{
Micaela Ribeiro and Olga Sousa Carneiro \\ University of Minho, Department of Polymer Engineering, IPC/I3N, Institute for Polymers and Composites, Guimarães, Portugal, and \\ Alexandre Ferreira da Silva \\ CMEMS-UMinho - Center of Electro Mechanical Systems, University of Minho, Guimarães, Portugal
}

\begin{abstract}
Purpose - An issue when printing multi-material objects is understanding how different materials will perform together, especially because interfaces between them are always created. This paper aims to address this interface from a mechanical perspective and evaluates how it should be designed for a better mechanical performance.

Design/methodology/approach - Different interface mechanisms were considered, namely, microscopic interfaces that are based on chemical bonding and were represented with a U-shape interface; a macroscopic interface characterized by a mechanical interlocking mechanism, represented by a T-shape interface; and a mesoscopic interface that sits between other interface systems and that was represented by a dovetail shape geometry. All these different interfaces were tested in two different material sets, namely, poly (lactic acid)-poly (lactic acid) and poly (lactic acid)-thermoplastic polyurethane material pairs. These two sets represent high- and low-compatibility materials sets, respectively.

Findings - The results showed, despite the materials' compatibility level, multi-material objects will have a better mechanical performance through a macroscopic interface, as it is based on a mechanical interlocking system, of which performance cannot be achieved by a simple face-to-face interface even when considering the same material.

Originality/value - The paper investigates the importance of interface design in multi-material 3D prints by fused filament fabrication. Especially, for parts intended to be subjected to mechanical efforts, simple face-to-face interfaces are not sufficient and more robust and macroscopic-based interface geometries (based on mechanical interlocking systems) are advised. Moreover, such interfaces do not raise esthetic problems because of their working principle; the 3D printing technology can hide the interface geometries, if required.
\end{abstract}

Keywords Fused deposition modelling, Additive manufacturing, 3D printing, Multimaterials, Interface

Paper type Research paper

\section{Introduction}

With the evolution of additive manufacturing (AM) and $3 \mathrm{D}$ printing technologies within AM, today it is not only possible to materialize virtual or mental concepts in a short period but also to fabricate personalized parts in small series. The impact of this set of technologies has been quite expressive, with continuous equipment upgrades coming out featuring increasing number of functionalities and new materials possibilities (Le Duigou et al., 2016). Srivastava (2017) made a recent review on the advances in the manufacturing of functional parts by $3 \mathrm{D}$ printing techniques. Not only is the number of materials and techniques identified is large but also the industrial sectors taking advantage of such techniques is increasing (namely, automotive, pharma or retail). As a result, many developments at the material level and studies regarding technique parameters' influenced in the part quality are emerging.

The current issue and full text archive of this journal is available on Emerald Insight at: www.emeraldinsight.com/1355-2546.htm

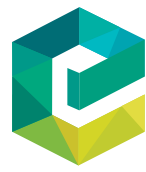

Rapid Prototyping Journal

25/1 (2019) 38-46

(C) Emerald Publishing Limited [ISSN 1355-2546]

[DOI 10.1108/RPJ-05-2017-0107]
Among the 3D printing techniques, fused filament fabrication (FFF) is currently the one with the highest growth rate and impact. This technique combines simplicity with low cost and increasing range of available materials (Tsouknidas et al., 2016). The potential of such manufacturing process is such that it has been used in combination with others in hybrid approaches to further develop current manufacturing possibilities (Ma et al., 2015). Hybrid manufacturing has been explored by combining additive (3D print) with subtractive as computer numerical control machining manufacturing processes. In the case of Ma et al. (2015), different additive processes as FFF and material deposition combined with components embedment were put together to push AM to new levels of capabilities (less assembly and complexity). FFF has been proving the potential to rethink how objects can be manufactured more efficiently.

Although FFF's initial range of material was barely limited to acrylonitrile butadiene styrene, PLA and polyvinyl alcohol

The authors acknowledge the funding by FCT with the reference projects UID/EEA/04436/2013 and UID/CTM/50025/2013, by FEDER funds through the COMPETE 2020 - Programa Operacional Competitividade e Internacionalização (POCI) with the reference project POCI-01-0145FEDER-006941.

Received 31 May 2017

Revised 18 September 2017

Accepted 26 January 2018 
polymers, today the set of materials is wider, including nonconventional ones such as flexible, phosphorescent, electric conductors, magnetic, metallic or wooden-filled filaments, among many others.

Such availability of materials has unlocked a set of possibilities regarding the design of parts, as the maker can now select the most suitable material for the purpose of the object being printed (depending on if it is for esthetics, mechanical, thermal or electrical purposes, for example).

Moreover, with such vast range of materials, it is now possible to produce multi-material components in such a way that a single object can be made from different materials at different locations that have different requirements. Looking back at conventional plastics manufacturing processes, a similar evolution occurred with injection molding, where today one can find multi-material injection molding, for example (Park and Lee, 2016). Thus, this extended the capabilities of the FFF process, making it a competitive manufacturing process, especially for small series production, where injection molds fabrication is economically unfeasible and multimaterial injection molding machines are still considered nonconventional equipment and require the use of several molds. The fabrication of multi-material parts by FFF is a technical and cost-efficient challenge that brings a range of possibilities. This becomes clearer when one thinks on using multi-material parts to reduce and to eliminate assembling processes and to minimize the number of parts that constitute a given component.

With such potential and interest, a few studies that explore the possibilities of multi-material $\mathrm{FFF}$ and process tuning have caught attention. As an example, Espalin et al. (2012) and Espalin et al. (2014) designed and built a two-material FFF system with a customized controlled software to demonstrate the benefits of variable layer thickness and raster width in the surface roughness. This had positive effects on the quality of the print, as the authors showed, by being able to reduce the roughness by $38-55$ per cent and also to reduce the print time by 53 per cent. These are relevant figures when seeking to propose $\mathrm{FFF}$ as an alternative manufacturing process.

Nonetheless, still most of the literature information focus mainly on a single material, and even so, it is scarce (Tymrak et al., 2014; Casavola et al., 2016) and mainly based on printing operating window (namely, print temperature and velocity). Moreover, in multi-material designs and prints, there are other specific features and parameters to be considered. From the start, there is a chemical compatibility issue, as in multimaterial prints different materials share a small percentage of overlap that defines this particular interface area. Depending on the compatibility level, one might end with a stronger or weaker interface, which then translates to the part's performance.

Even so, one key advantage of $3 \mathrm{D}$ printing is the unconstrained geometry structure of the part, as it is built layerby-layer. Thus, the interface between different materials can be designed (and even become non-visible on the final part) to promote the best interconnection (interlocking) between them.

This paper aims at studying the interface geometries (between dissimilar materials) considering a multi-material print from two high- and low-compatible materials' sets. To evaluate this interface performance, test specimens were printed by FFF and tested in a tensile testing machine.

\section{Joints geometries}

In comparison with single material parts, multi-material objects have a key characteristic in their design, its interface between different materials, as they need to be coupled without the use of fixation aid/elements (welding, adhesives, screws, etc.). When dealing with multi-material objects/parts, two possible situations can occur at this interface (Figure 1), the object has either a graded transition or a discrete transition between the different materials. At first, there is no clear limit or interface that separates different materials but instead a continuous gradient transition of composition between both. On the discrete transition, a clear interface is defined, which bounds both materials.

In $3 \mathrm{D}$ printing by $\mathrm{FFF}$, it is not yet possible to achieve a gradient transition because of the process working principle. Therefore, in multi-material prints, the interfaces that will result between different materials are discrete and well defined, as they result from the transition between extrusion heads or material filament change. Within the discrete interface scenarios, different mechanisms (Figure 2) can be defined, namely, macroscopic, microscopic and mesoscopic (Fowler, 2004).

Microscopic interfaces [Figure 2(a)] are known as chemical interfaces and result from chemical bonding along coupled surfaces between different materials. This mechanism is highly dependent on the molecular nature and architecture of the materials involved, their viscosities and processing temperatures. As expected, this type of interface is more prone to exist between materials that have a high level of compatibility or chemical affinity.

On the other side of the interface spectrum, there are macroscopic interfaces [Figure 2(b)]. In this case, the bond element between materials is based on a mechanical (geometric) structure or interlocking mechanism. These are interfaces more suitable for materials with low level of compatibility.

Figure 1 Interface scenarios

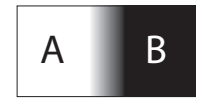

(a)

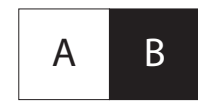

(b)
Notes: (a) Graded interface;

(b) discrete interface

Figure 2 Discrete interface mechanisms

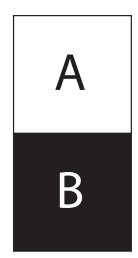

(a)

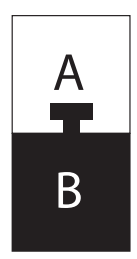

(b)

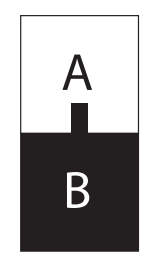

(c)
Notes: (a) Microscopic; (b) macroscopic; (c) mesoscopic 
The mesoscopic interface [Figure 2(c)] positions itself between the others, presenting an interface mechanism through chemical bonding and mechanical interlocking.

These interfaces might be improved with additional elements, if necessary, like adhesion enhancers along the interface. In this case, it can be thought as using an additional material aiming to promote a good chemical bond between two incompatible materials. This is a common solution used in the production of technical co-extruded films (Schrenk and Alfrey, 1978).

From a mechanical point of view, interfaces are many a time considered as weak points of a structure, as they represent a discontinuity along it. Having in mind the aforementioned, this work aims at assessing the performance of the interface formed between dissimilar materials in multi-material prints by FFF.

\section{Materials and methods}

In this section, the methodology used in this study, the interface geometries considered, the printing conditions used and the mechanical characterization of the printed samples are described in detail.

\subsection{Methodology}

The performance criterion selected in this study was mechanical analysis. Despite of different types of interfaces (microscopic, mesoscopic or macroscopic), all of them target the structural integrity of a given part. Therefore, a mechanical test of the part provides relevant data about the interface performance.

The process started by defining a set of interface geometries that would bring the most of the different interface mechanisms presented before. These geometries were then printed in the shape of a test specimen for tensile mechanical tests.

Furthermore, these test specimens were printed in multimaterial scheme with a pair of materials that ensured a high and a low compatibility level. This enabled to assure that both the mechanical and chemical bonding between different materials would be tested.

The interface performance was analysed and discussed according to the Young's modulus, ultimate (maximum) strength and strain at break.

\subsection{Interface designs}

Figure 3 shows different interface geometries considered in the study. The goal is to target geometries that present a prominent interface mechanism (microscopic, macroscopic and mesoscopic).

In the first geometry [Figure 3(a)], the interface depends on a recess element, a T-shape element. This is, in essence, a purely mechanical interface mechanism with a clear interlocking system. Thus, in this case, a mechanical interface (macroscopic) is obtained.

Figure 3(b) illustrates the simplest interface case with a Ushape connection, or tongue and groove. In this geometry, there is no true interlocking mechanism (from an axial tensile load perspective), and therefore, the interface performance is highly dependent on the chemical affinity between the two materials.

The mesoscopic interface geometry [Figure 3(c)] results from a simplification of macroscopic interface, where the locking mechanism was simplified (and reduced), but the
Figure 3 Tested interface geometries
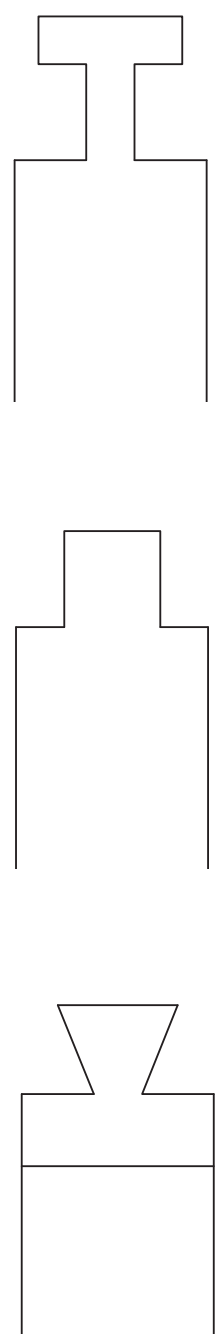

Notes: (a) T-shape; (b) U-shape; (c) dovetail shape contact area between the two materials is higher than that corresponding to the microscopic geometry. This enabled the interface design to be positioned between the other two designs in terms of mechanical-chemical bond mechanism. The overall design of this geometry is similar to a dovetail shape.

\subsection{Filaments materials}

Two different materials were sought to enable a pair of highand low-compatible materials.

For the high-compatible materials, poly (lactic acid)-poly (lactic acid) (PLA-PLA) pair was selected. PLA is possibly the most widespread filament material used in FFF.

By having the same material in both parts of the test specimen, it is possible to get the highest compatibility level from a chemical perspective. In this case, black PLA filament with 1.75 -mmdiameter with reference PLA-BSC-BLACK-175 was used.

On the other hand, to provide a low level of material compatibility, the pair poly (lactic acid)-thermoplastic 
polyurethane (PLA-TPU) was defined. TPU is an elastomer with high level of elasticity (Bates et al., 2016) and presents low affinity with PLA from a chemical perspective.

Finally, it should be noted that by using PLA in both pairs, it was possible to establish a reference material for proper results comparison.

\subsection{Printing conditions}

All test specimens were printed in a Prusa i3 printer (Carneiro et al., 2015) modified for multi-material printing. The extrusion system was based on two direct drive Heatcore Unibody extrusion heads from BQ, being compatible with many filament materials including the ones considered in this study. Table I shows major printing conditions used to fabricate the test specimens, and Figure 4 illustrates the schematic of the FFF tool used.

\subsection{Tensile tests}

The test specimens were mechanically tested under tensile loads to evaluate the interface geometries performance.

The tensile test specimens were printed according to the nominal dimensions in the standard DIN 53504-S2a (Figure 5).

The tensile tests were performed in a ZWICK equipment at a cross-head speed of $50 \mathrm{~mm} / \mathrm{min}$, with a $20-\mathrm{mm}$ grip distance and at room temperature. For each condition, five test specimens were tested.

Table I Printing conditions

\begin{tabular}{lcl}
\hline Property & Value & Unit \\
\hline Layer thickness & 0.2 & $\mathrm{~mm}$ \\
Shell thickness & 1.2 & $\mathrm{~mm}$ \\
Density & 100 & $\%$ \\
Orientation & \pm 45 & $\circ$ \\
Print speed & 20 & $\mathrm{~mm} / \mathrm{s}$ \\
Bed temperature & 60 & ${ }^{\circ} \mathrm{C}$ \\
Filament diameter & 1.75 & $\mathrm{~mm}$ \\
Flow & 100 & $\%$ \\
Nozzle diameter & 0.4 & $\mathrm{~mm}$ \\
PLA temperature & 200 & ${ }^{\circ} \mathrm{C}$ \\
TPU temperature & 225 & ${ }^{\circ} \mathrm{C}$ \\
\hline
\end{tabular}

Figure 4 Schematic of the used FFF tool

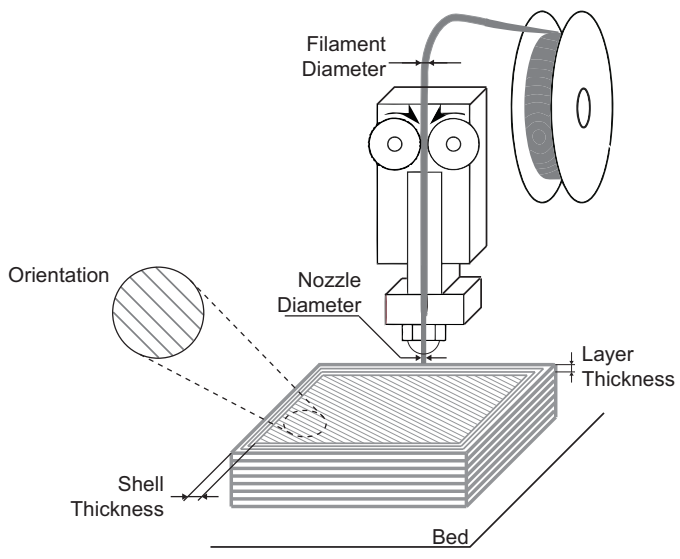

Figure 5 Tensile test specimen's dimensions (values in millimeters)

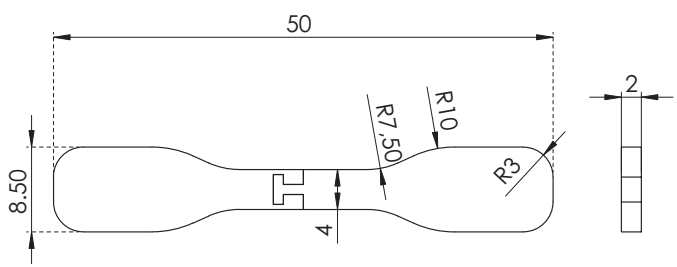

\section{Results and discussion}

In this section, the test results are presented and discussed. For a better interpretation of the results, they were divided into material pair set, being the results evaluated based on the material pair and interface geometry.

\subsection{Print quality}

A critical detail in FFF technology is the creation of the print file, which according to the parameters defined in the slicer software define the print procedure, namely, raster orientation.

Figure 6 details, for different interface shapes considered in the study, how the filament raster was printed. One can easily identify three outline perimeters that define the shell thickness of

Figure 6 Print raster's details for the different interface shapes
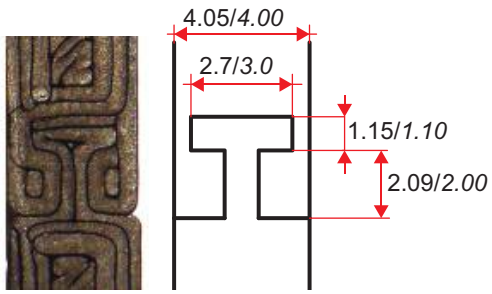

(a)
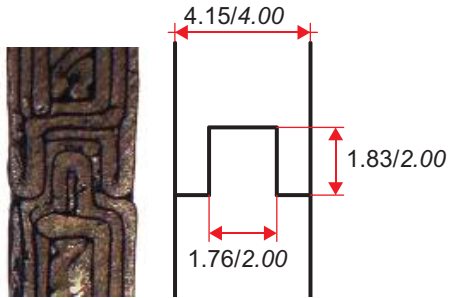

(b)
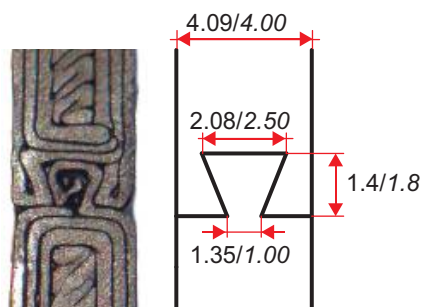

(c)

Notes: (a) T-shape; (b) U-shape; (c) Dovetail shape. All dimensions values are shown in the following format: Print/CAD [mm] 
$1.2 \mathrm{~mm}$ (as stated in Table I), based on the nozzle size of $0.4 \mathrm{~mm}$. Despite the pair of material considered, the filament deposition layout was the same, as the layout is defined not by the material properties but by the part's design and printing conditions.

Based on the shown dimensions, one can infer regarding the print quality in respect to the geometric features. There are deviations from the computer-aided design (CAD) file dimensions, varying in the range from 1.25 to 22 per cent. As the print process started by defining the part's outer contour, the dimensions of the inside features, as they are small, end up being adjusted to the nozzle size and other print parameters (shell thickness, for example), resulting in larger dimensions deviations in some cases.

Overall, the different interface features are well outlined and defined in the test specimens, as all geometric details of the interfaces are easily identified.

\subsection{Rupture sites}

Before analysing the data results obtained from the tensile tests, a preliminary analysis can be performed to the test specimens' rupture sites.

Figures 7 and 8 show an example of a representative test specimen after being tested, as well as the illustration of the rupture line for the PLA-PLA and PLA-TPU material sets, respectively.

Looking only at the PLA-PLA material set test specimens (Figure 7), there is a clear rupture line that was only partially overlaying the materials' interface line. The results show a transverse continuous rupture across the test specimen, even across the geometric elements of the interface. This result suggests a strong bonding between parts, with sections of the interface being more robust than small outgoing features of the part.

Figure 7 Rupture sites illustration and photos of the PLA-PLA test specimens for the different interface geometries
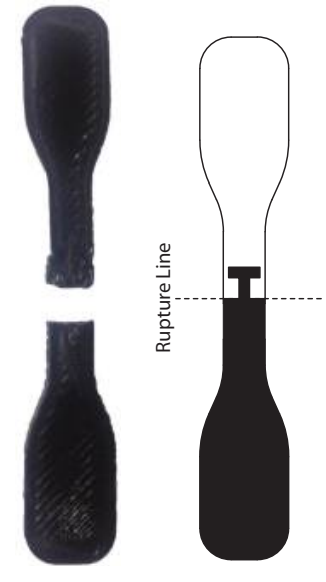

(a)

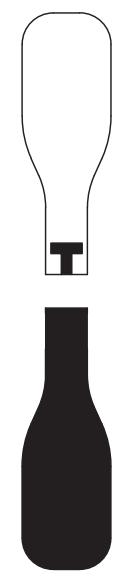

Notes: (a) T-shape; (b) U-shape; (c) Dovetail shape

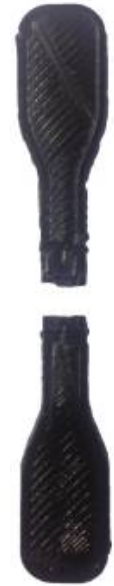

(b)
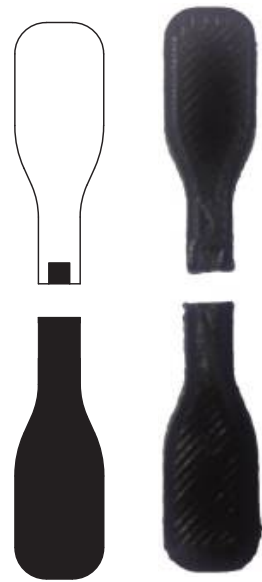

b)

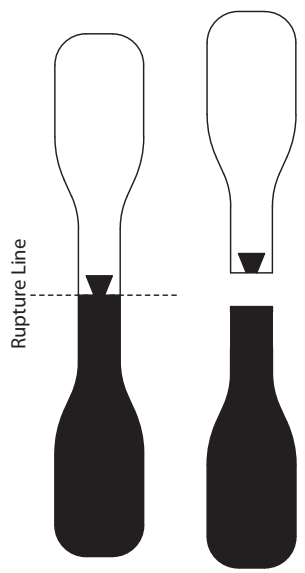

(c)

Figure 8 Rupture sites illustration and photos of the PLA-TPU test specimens for the different interface geometries

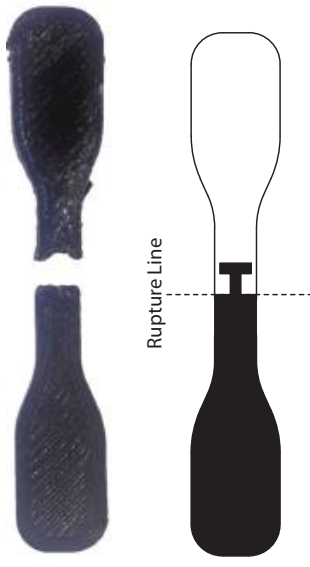

(a)
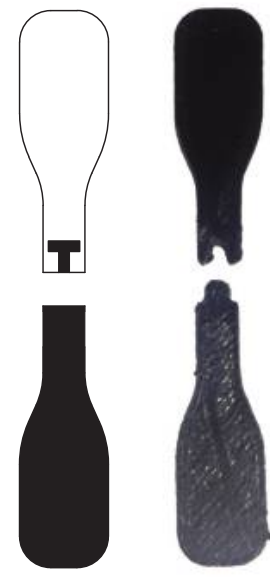

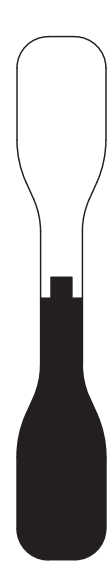

(b)
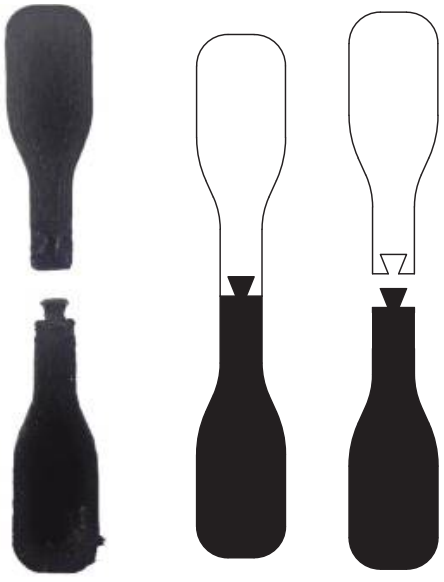

(c)

Notes: (a) T-shape; (b) U-shape; (c) Dovetail shape 
On the other side, the PLA-TPU material pair showed a slightly different result (Figure 8). Although the test specimens with the U-shape [Figure 8(b)] and dovetail shape [Figure 8 (c)] interface geometries resulted in a clear separation of both parts along the interface line (that slide over each other), T-shape shows a few remaining of similar behavior. Looking at Figure 8(b) and (c), as the test specimen was stretched and ruptured, the geometric features that represent the interface geometry were kept on its corresponding part. Compared to the PLA-PLA case, the material set PLA-TPU showed a clear evidence of low compatibility level, by having a detachment behavior.

T-shape, Figure 8(a), showed an in-between behavior from what was seen in the PLA-PLA case and in the PLA-TPU for the U-shape and dovetail shape case. The complete features of the interface are not present at the end, but some levels of remaining are, namely, the female part of the interface. Thus, in this case, despite being a low-compatibility material set, the T-shape interface showed the capability of interlocking both parts of the test specimen during the test, resulting in the breakage of the outgoing geometry.

Combining the results illustrated in Figures 7 and 8, jointly with the print raster's details illustrated in Figure 6, one has data to infer about the interaction between filament raster and part performance results. In Figure 8(b) and (c), the interface features as the pin/U-structure shape or the dovetail details were still connected to its respective part after the test specimen rupture. This suggests that few filaments that outline the interface features and link it to the body part of the test specimen are structurally well printed and deposited in each layer. If this was not the case, then, all the results would be similar to the ones presented in Figure 7, with the test specimen breaking across the interface geometric features. Thus, although there is a certain level of anisotropy inherent to the FFF process driven by the filament deposition layout, overall the parts and the interface geometric features were successfully fabricated and the interface performance became highly dependent on the interface geometry.

\subsection{Mechanical properties of poly (lactic acid)-poly (lactic acid) specimens}

Figure 9(a) illustrates the Young's modulus obtained for the PLA-PLA test. Overall, one easily verifies that most of the determined values are within the same range and order level, despite different interface geometries used in the specimens.

The PLA-PLA pair was selected to provide best compatibility between materials, which would be expected to be the highest, if using the same material. Thus, in this test, the variations of Young's moduli are mainly driven by the interface performance, and despite the low level of variation, it is still worth to further analyse them.

It is a clear understanding that the existence of interfaces represents a weak point on the structure. This is considered valid, as interfaces are physical discontinuities/transitions in a given structure. Focusing on the Young's modulus, the T-shape interface geometry is the one that presents the highest value among the tested interfaces, being also the one with the highest tensile stress [Figure 9(b)]. Such performance figures

Figure 9 PLA-PLA test specimens tensile test results

(a)

(b)

(c)

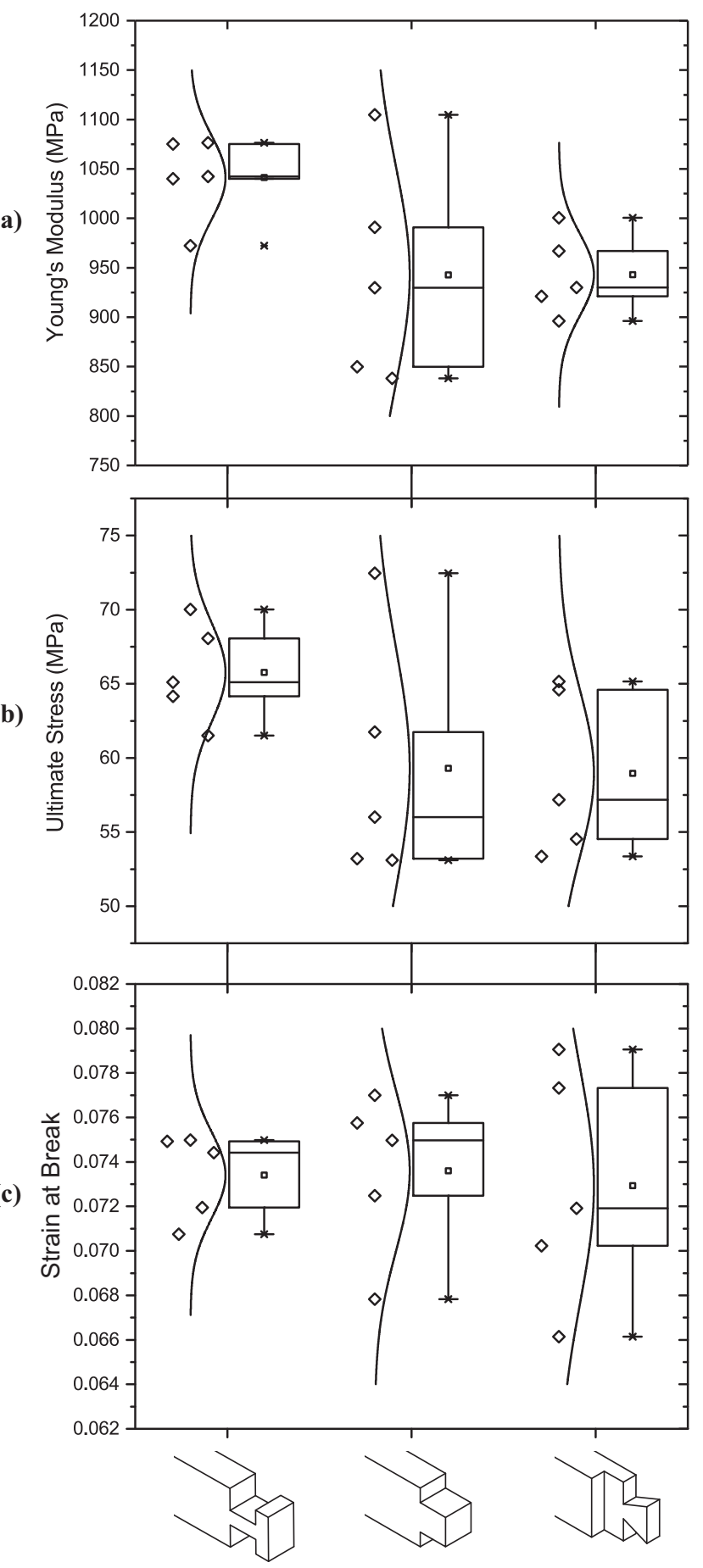

Notes: (a) Young's modulus; (b) ultimate stress; (c) strain at break

are clearly related to the interface interlocking mechanism. It represents a true mechanical system, which seems to survive longer than the other interfaces that are more prone to chemical bonding.

The interface with U-shape is mainly based on chemical bond. Even considering the side walls, there is no significant 
mechanical constriction over the center pin element in one of the parts of the test specimen and its negative counterpart. Both parts are connected by a small overlap value, and by a chemical connection between both component materials. Therefore, looking at the result, one clearly identifies a drop in the Young's modulus.

The dovetail shape Young's modulus figures are closer to those of the U-shape geometry. It presents a mechanical locking mechanism that is not as strong as $\mathrm{T}$-shape but that still enables a certain degree of mechanical bonding. Similarly, to T-shape, dovetail shape ensured a low level of variability, with most test specimens performing equally.

Using the Young's modulus analysis as the unique criterion becomes short for the complete assessment of the interface geometry performance. Therefore, adding the ultimate stress and the strain at break, a deeper understanding is possible.

Looking at the overall results with the different elements (Figure 9), U-shape was the one presenting, in average, the highest maximum strain. Such result is derived from the geometry shape itself, which enables both parts of the test specimen to slide out, in opposition to what happens in the T-shape interface that, when undergoing tensile strain, promotes the straining and rupture of the neck at the T section, which leads to the highest Young's modulus. In opposition, Ushape is not based on a mechanical interlocking mechanism but can be seen as a simplified insert feature, enabling both parts of the test specimen to separate by sliding out, resulting in the highest strain at break.

For dovetail shape, there is an in-between behavior, as the interlocking mechanism is not as strong as T-shape. When subjected to tensile efforts, the lateral sides of the dovetail force the claw elements of the other part to open until breakage. Moreover, there is a large interface area subjected to shear.

Such type of behavior suggests that despite both parts being made of the same material, the interface performance is more dependent on the macroscopic level (mechanical interlock) than on the microscopic one (chemical bonding). The possibility for a slide behavior at the interface represents a failure of chemical bonding, as it is accompanied by a lowering of the tensile stress.

\subsection{Mechanical properties of poly (lactic acid)- thermoplastic polyurethane sets}

Looking at the PLA-TPU material pair (Figure 10), an overall drop in the Young's modulus and ultimate stress for all geometries is identified. Such a behavior was already expected because of TPU's material nature.

From the Young's modulus [Figure 10(a)] perspective, the PLA-TPU material pair follows a similar behavior to the previous analysed PLA-PLA one. There continues to exist signs that interface geometries that present a mechanical interlocking mechanism are the ones that result in a higher modulus, mainly driven by the mechanical interlocking.

Looking at the tensile test results from the strain at break perspective [Figure 10(c)], the behavior observed is different from that of PLA-PLA case. For this material pair, the higher strain at break is achieved with the T-shape interface geometry. This behavior clearly highlights the TPU's elastic capability. By locking the two elements of the test specimen, it became

Figure 10 PLA-TPU test specimens tensile tests results

(a)

(b)

(c)

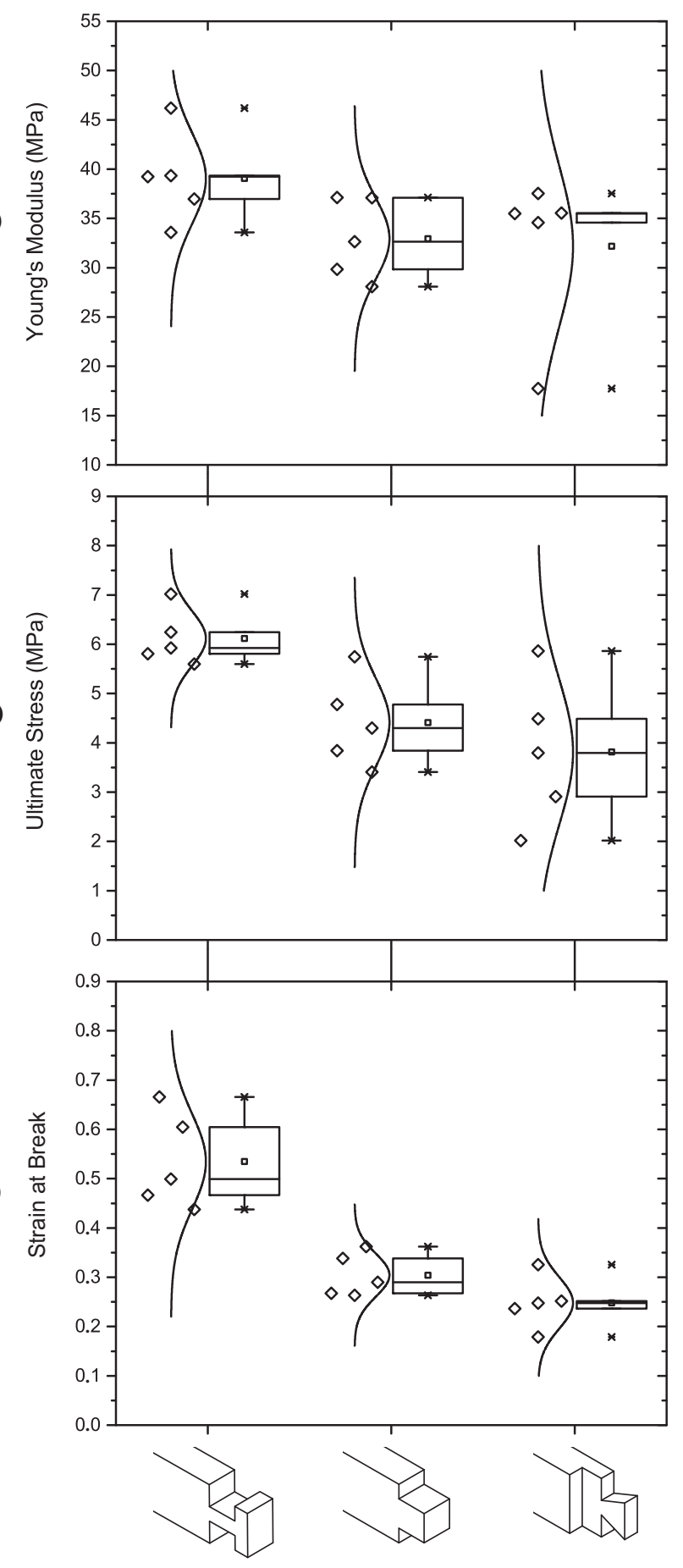

Notes: (a) Young's modulus; (b) ultimate stress; (c) strain at break

possible to attain larger deformations through the TPU element, without breakage or sliding off, resulting in the highest maximum strain.

On the other hand, in the U-shape case as soon as the TPU is elongated, and as the interface is located at the site subjected to the highest stress, it breaks loose from the interface, resulting in a lower strain at break at a lower level 
of stress. This point outs a mechanism that is more dependent on chemical bonding and that enables a slide out when such interaction vanishes.

Such a behavior is distinctive from the PLA-PLA material set because of the inherent Young's modulus of PLA versus TPU.

Looking back at the PLA-PLA specimens, one has a rigid material, which therefore tends to present a brittle rupture. As a result, the interface geometries that force a strong interlocking result in higher Young's modulus values, corresponding to lower elongation and high levels of stress at break.

In the TPU case, one shifted from a brittle to a ductile-like behavior. As a result, the interface with the strongest locking mechanism simulates a kind of claw system over the TPU element, which enables it to be drawn further.

Thus, from an interface design perspective, a macroscopic interface, i.e. based on a mechanical interlocking, enables better interface mechanical performance, resulting in a behavior that tends for a less rigid material component part.

\section{Conclusion}

This paper evaluated the interface mechanism for multimaterial 3D prints. This is a critical element when designing these type of parts, as multi-material printing systems enable multi-material objects to be printed in a single print run. However, the existence of different material sections results in the creation of interfaces between those materials, which from a mechanical perspective, represent potential weak zones of the objects, especially critical when they are subjected to mechanical loads.

By defining a set of different geometry interfaces between different materials that represent different bonding mechanisms, from microscopic (chemical) to macroscopic (mechanical), it was possible to evaluate how they behave with high-compatibility (PLA-PLA) and low-compatibility (PLATPU) material sets.

From the obtained results under tensile tests, it was verified that between microscopic and macroscopic interfaces, the latter one is the one providing a better mechanical performance. Despite using the same material to guarantee the highest compatibility, the FFF process as it occurs, does not promote a uniform structure and a weak zone still exist at the interface. Possible reasons for this effect are mainly related to deposition (printing) layout, which creates a physical boundary between the two bodies (despite being printed with the same material). Moreover, as a possible additional driver to promote the structure non-uniformity, is the printing sequence that, depending on the object geometry, might result in long durations between continuous sections print, and therefore, the best conditions to promote polymer sintering might not be accomplished. This latter issue should be considered in followup research.

Furthermore, it is important to point out the quality of the print, as it was shown; there were some deviations on the part dimensions versus the dimensions in the CAD file. Despite these differences and other conditions that might infer with the printed part, the results can be evaluated in a comparative manner between interface designs, which still demonstrates a trend behavior of the interface mechanism.
Overall, this work showed that when dealing with multimaterial parts, if they are intended to be subjected to mechanical efforts, a simple face-to-face interface (or so-called butt joint, in welding technology) might not have enough resistance, and therefore, more robust and macroscopic-based interface geometries (based on mechanical interlocking systems) are advised. This does not raise esthetic problems because of its working principle; the $3 \mathrm{D}$ printing technology enables to hide the interface geometries, if required.

\section{References}

Bates, S.R.G., Farrow, I.R. and Trask, R.S. (2016), "3D printed polyurethane honeycombs for repeated tailored energy absorption", Materials and Design, Vol. 112, pp. 172-183, available at: http://dx.doi.org/10.1016/j. matdes.2016.08.062

Carneiro, O.S., Silva, A.F. and Gomes, R. (2015), "Fused deposition modeling with polypropylene", Materials and Design, Vol. 83, pp. 768-776, available at: http://dx.doi.org/ 10.1016/j.matdes.2015.06.053

Casavola, C., Cazzato, A., Moramarco, V. and Pappalettere, C. (2016), "Orthotropic mechanical properties of fused deposition modelling parts described by classical laminate theory", Materials and Design, Vol. 90, pp. 453-458, available at: http://dx.doi.org/10.1016/j.matdes.2015.11.009

Espalin, D., Ramirez, J., Medina, F. and Wicker, R., (2012), "Multi-material, multi-technology FDM system", in SFF Symposium, Austin, TX, pp. 828-835.

Espalin, D., Alberto Ramirez, J., Medina, F. and Wicker, R. (2014), "Multi-material, multi-technology FDM: exploring build process variations", Rapid Prototyping fournal, Vol. 20 No. 3, pp. 236-244, available at: http://dx.doi.org/10.1108/ RPJ-12-2012-0112

Fowler, G.T. (2004), Cost and Performance Evaluation Models for Comparing Multi-Shot and Traditional Injection Molding, University of MD, available at: http://drum.lib.umd.edu/ handle/1903/1759

Le Duigou, A., Castro, M., Bevan, R. and Martin, N. (2016), "3D printing of wood fibre biocomposites: from mechanical to actuation functionality", Materials and Design, Vol. 96, pp. 106-114, available at: http://dx.doi.org/10.1016/j. matdes.2016.02.018

Ma, R.R., Belter, J.T. and Dollar, A.M. (2015), "Hybrid deposition manufacturing: design strategies for multimaterial mechanisms via three-dimensional printing and material deposition", fournal of Mechanisms and Robotics, Vol. 7 No. 2, p. 21002, available at: http://dx.doi.org/ 10.1115/1.4029400

Park, S.-J. and Lee, S.-Y. (2016), "Injection molding for multicomponent materials", in Kim, J.K., Thomas, S. and Saha, P. (Eds), Multicomponent Polymeric Materials, Springer, Berlin, pp. 79-107, available at: https://dx.doi.org/10.1007/ 978-94-017-7324-9_4

Schrenk, W.J. and Alfrey, T. Jr (1978), “Coextruded multilayer polymer films and sheets", in Paul, D.R. and Newman, S. (Eds), Polymer Blends, Vol. 2, pp. 129-165.

Srivastava, V.K. (2017), "A review on advances in rapid prototype 3D printing of multi-functional applications", Science and Technology, Vol. 7 No. 1, pp. 4-24. 
Tsouknidas, A., Pantazopoulos, M., Katsoulis, I., Fasnakis, D., Maropoulos, S. and Michailidis, N. (2016), "Impact absorption capacity of 3D-printed components fabricated by fused deposition modelling", Materials and Design, Vol. 102, pp. 41-44, available at: http://dx.doi.org/10.1016/j. matdes.2016.03.154

Tymrak, B.M., Kreiger, M. and Pearce, J.M. (2014), "Mechanical properties of components fabricated with open-source 3-D printers under realistic environmental conditions", Materials and Design, Vol. 58, pp. 242-246, available at: http://dx.doi.org/10.1016/j.matdes.2014.02. 038

\section{Corresponding author}

Alexandre Ferreira da Silva can be contacted at: asilva@, dei.uminho.pt

For instructions on how to order reprints of this article, please visit our website:

www.emeraldgrouppublishing.com/licensing/reprints.htm

Or contact us for further details: permissions@emeraldinsight.com 